\title{
Implementation of Health Insurance Support Tools in Community Health Centers
}

\author{
Nathalie Huguet, PhD, Brigit Hatch, MD, MPH, Aleksandra Sumic, MPH, \\ Carrie Tillotson, MPH, Elizabeth Hicks, MPH, Joan Nelson, PA-C, and \\ Jennifer E. DeVoe, MD, DPhil
}

Background: Health information technology (HIT) provides new opportunities for primary care clinics to support patients with health insurance enrollment and maintenance. We present strategies, early findings, and clinic reflections on the development and implementation of HIT tools designed to streamline and improve health insurance tracking at community health centers.

Methods: We are conducting a hybrid implementation-effectiveness trial to assess novel health insurance enrollment and support tools in primary care clinics. Twenty-three clinics in 7 health centers from the OCHIN practice-based research network are participating in the implementation component of the trial. Participating health centers were randomized to 1 of 2 levels of implementation support, including arm $1(n=4$ health centers, 11 clinic sites) that received HIT tools and educational materials and arm 2 ( $n=3$ health centers, 12 clinic sites) that received HIT tools, educational materials, and individualized implementation support with a practice coach. We used mixed-methods (qualitative and quantitative) to assess tool use rates and facilitators and barriers to implementation in the first 6 months.

Results: Clinics reported favorable attitudes toward the HIT tools, which replace less efficient and more cumbersome processes, and reflect on the importance of clinic engagement in tool development and refinement. Five of 7 health centers are now regularly using the tools and are actively working to increase tool use. Six months after formal implementation, arm 2 clinics demonstrated higher rates of tool use, compared with arm 1.

Discussion: These results highlight the value of early clinic input in tool development, the potential benefit of practice coaching during HIT tool development and implementation, and a novel method for coupling a hybrid implementation-effectiveness design with principles of improvement science in primary care research. (J Am Board Fam Med 2018;31:410-416.)

Keywords: Community Health Centers, Health Insurance, Implementation Science, Medical Informatics, Mentoring, Primary Health Care

Health insurance is critically important for access to health care services. Compared with insured patients, uninsured patients have higher rates of unmet health care needs, receive fewer preventive services, and are more likely to visit emergency

This article was externally peer reviewed.

Submitted 4 July 2017; revised 26 August 2017; accepted 31 August 2017.

From Oregon Health \& Science University, Portland, OR (NH, BH, EH, JED); OCHIN, Inc, Portland (BH, AS, CT, JN, JED).

Funding: This study was supported by National Cancer Institute R01CA181452.

Conflict of interest: none declared.

Corresponding author: Brigit Hatch, MD MPH, Oregon Health and Science University, 3181 SW Sam Jackson Park Rd, Portland, OR 97239 (E-mail: adamusb@ohsu.edu). departments to address conditions treatable in primary care. ${ }^{1-8}$ As a result, uninsured patients are at increased risk for preventable morbidity and mortality. ${ }^{9,10}$

In 2014, implementation of the Patient Protection and Affordable Care Act greatly expanded access to health insurance coverage in the United States ${ }^{11-13}$, and the number of uninsured Americans dropped from 43 million in 2013 to 27 million in 2016. ${ }^{14}$ Although the Patient Protection and Affordable Care Act increased opportunities for patients to obtain coverage in 2014, many continue to experience difficulties navigating the complex systems of health insurance enrollment and maintenance. ${ }^{15}$ To address this problem, the Health 
Resources and Services Administration (HRSA) provides grants to health centers for patient assistance in gaining and maintaining insurance. Over 1000 community health centers (CHCs) have been awarded these grants. ${ }^{16}$ In CHCs, health insurance eligibility specialists assist patients with enrolling and re-enrolling in health insurance, especially with Medicaid. Patients needing assistance may self-identify, be referred at the time of an appointment, or identified for outreach outside of the context of an appointment. Most outreach and enrollment (O\&E) efforts remain difficult to track and are largely limited to self-selected patients in the context of an office visit. Recent advances in health information technology (HIT) provide opportunities for developing HIT tools to facilitate more timely and effective support for health insurance enrollment and maintenance.

Our team developed a suite of health insurance support tools integrated within the electronic health record (EHR). The tools consist of an electronic "form," which appears alongside typical patient registration processes within the EHR. This form, intended for use by eligibility specialists or other staff who assist patients with registration and insurance enrollment, includes discrete fields for documenting important insurance enrollment information such as the status of a patient's insurance application, insurance identification, and effective date. The tools also include a reporting function to identify patients who do not have coverage so that needed outreach can be provided to these individuals. The tools were designed to streamline and improve health insurance tracking at $\mathrm{CHCs}$, with the aim of increasing insurance continuity, reducing uninsured visits, and improving patient care.

Using principles of improvement science and a hybrid effectiveness-implementation research design, we aimed to implement these tools at primary care clinics within the OCHIN network and study their uptake, use, and effectiveness. In this ongoing study, we present early findings in tool use and preliminary qualitative reflections from clinics actively participating in the implementation.

\section{Methods}

\section{Study Design}

This study has a hybrid effectiveness-implementation design, as depicted in Figure 1 and described by Curran and collaborators ${ }^{17}$, which allows simul-
Figure 1. Hybrid effectiveness-implementation study design.

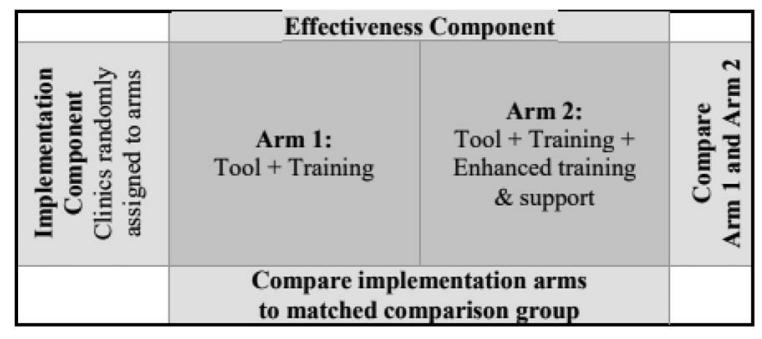

taneous assessment of the effectiveness of our health insurance support HIT tool and the best strategy for implementing the tool in CHCs. Health centers recruited for tool implementation were randomized to 1 of 2 levels of implementation support; arm 1 clinics received the tool and educational materials only and arm 2 clinics contributed to tool testing and refinement, received the tool and educational materials, and are receiving ongoing individualized implementation support with a practice coach.

\section{Study Setting and Population}

Implementation clinics were recruited from the OCHIN practice-based research network (PBRN). ${ }^{18}$ OCHIN is the largest network of CHCs using a single instance of the Epic EHR. Its centrally hosted Epic EHR is deployed in nearly 100 organizations caring for nearly 2,000,000 patients across 18 states. Participating health centers were recruited from a subset of OCHIN PBRN members meeting the following criteria: location in a state that expanded Medicaid in 2014, implementation of the OCHIN EHR before 2013, and 1,000 adult patients with one visit in the year before the study. Twenty-three clinics in 7 health centers are participating in tool implementation, ranging from 1 to 6 clinics per health center. Most health centers are designated as Federally Qualified Health Centers. All participating clinics received HRSA grant funding for outreach and enrollment and are located in Oregon, California, and Ohio. Four health centers (11 clinic sites) were randomized to arm 1; 3 health centers ( 12 clinic sites) were randomized to arm 2. The 23 implementation sites were propensity score matched to 23 control clinic sites that are contributing data, but will not be receiving the intervention. ${ }^{19}$ This article reports data from the 23 implementation clinics; it does not report on the 
23 control clinics, because these CHCs did not participate in the intervention and did not have access to the HIT tools. Data from the control clinics will be used to assess tool effectiveness at the conclusion of the study period.

\section{Study Period}

Study phase 1 consisted of a 6-month tool testing and refinement window (March to September 2016) where the initial version of the tools was released to arm 1 and arm 2 clinics. Arm 2 clinics were engaged in " $\beta$ testing" the tools and participated in user-centered design feedback sessions that led to tool refinement and updates. Study phase 2 commenced with implementation of the final version of the tools in mid-September 2016. This second phase of the study is planned to be 18 months (through March 2018); early results presented here include data through May 2017, as the study is ongoing.

\section{Implementation Support}

Implementation support for arm 2 clinics is customized to meet individual practice needs. A single practice coach was assigned to arm 2 clinics to facilitate early $\beta$ testing and provide longitudinal implementation support. The practice coach visited each arm 2 health center during the tool development phase and received feedback on all aspects of the tools, including an assessment of facilitators and barriers to their implementation. During the second phase of the study (after implementation of the updated tools), the practice coach is engaging in monthly contact with the clinics via web meetings, phone calls, tailored trainings, and/or email outreach, depending on the needs expressed by each clinic. During these fora, the practice coach is providing audit and feedback reports and tailored guidance to support clinic-led "plan-do-study-act" cycles focusing on enhancing tool use.

\section{Qualitative Methods}

In study phase 1 , qualitative data were collected through interviews and ethnographic observation from 2-day site visits, followed by monthly phone interviews with clinic informants from participating health centers. Interviews were audio recorded, professionally transcribed, and analyzed with grounded theory approach. ${ }^{20}$ Atlas.ti 7 software (Berlin) was used for data management and analysis. Qualitative data collection and analyses for study phase 2 is ongoing, so we are only able to report on early reflections and emerging themes.

\section{Quantitative Methods}

We are collecting clinic characteristics and tool use data throughout the study period (phases 1 and 2) via electronic data capture in the EHR. An instance of tool use is defined as the creation or update of an electronic enrollment support form. Tool use is being measured per health center on a monthly basis. We used SAS Version 9.4 (SAS Institute Inc., Cary, NC) to conduct analyses. The Institutional Review Board of the Oregon Health \& Science University has reviewed and approved this study. The study is registered with ClinicalTrials.gov (number NCT02355262).

\section{Results}

\section{Qualitative Findings}

During the tool development and refinement period (phase 1), 2 of the 3 arm 2 health centers were in the preliminary stages of conducting insurance "in-reach," contacting existing patients with upcoming appointments whose insurance was likely to expire in the next month. With the assistance of the implementation support coach, they had developed workflows to use the tool, trained staff, and were variably using the tool with patients. Staff expressed that in-reach efforts were important because, in comparison, prior efforts waited too long (often the day before an appointment) to let a patient know that insurance had expired. They reported that late identification of expired insurance caused stress and extra work for staff and often led to the patient's appointment not being covered by health insurance.

A staff member from health center $\mathrm{F}$ discussed challenges with patient notification of when Medicaid insurance periodically expires or "terms":

So that is why we really want to be on the front end of [insurance renewals]. Because when [the state] termed a whole bunch of people in October and a whole bunch of people in November, it was a nightmare because they did not send any letters. Nobody knew. So it was terrible. So this [tool] is going to be really, really cool. I am really excited about this.

Health center E already had an established outreach and enrollment tracking system in place for 
Table 1. Characteristics of Participating Health Centers

\begin{tabular}{|c|c|c|c|c|c|c|c|}
\hline Health center & A & B & $\mathrm{C}$ & $\mathrm{D}$ & $\mathrm{E}$ & $\mathrm{F}$ & G \\
\hline Type & FQHC & FQHC & FQHC & FQHC & FQHC & RHC & FQHC \\
\hline Number of patients & 3,523 & 18,263 & 3,761 & 3,826 & 12,507 & 3,654 & 19,151 \\
\hline \multicolumn{8}{|l|}{ Proportion of visits by payer } \\
\hline Uninsured & $6.4 \%$ & $11.9 \%$ & $11.5 \%$ & $45.4 \%$ & $5.5 \%$ & $6.5 \%$ & $10.4 \%$ \\
\hline Medicaid & $53.8 \%$ & $59.7 \%$ & $51.6 \%$ & $45.0 \%$ & $64.3 \%$ & $30.7 \%$ & $56.6 \%$ \\
\hline Medicare & $22.3 \%$ & $11.7 \%$ & $16.4 \%$ & $6.2 \%$ & $6.6 \%$ & $34.4 \%$ & $19.4 \%$ \\
\hline Commercially Insured & $15.7 \%$ & $16.5 \%$ & $18.2 \%$ & $1.5 \%$ & $2.9 \%$ & $27.5 \%$ & $13.2 \%$ \\
\hline Other public insurance* & $1.9 \%$ & $0.3 \%$ & $2.3 \%$ & $1.9 \%$ & $20.8 \%$ & $1.0 \%$ & $0.5 \%$ \\
\hline $\begin{array}{l}\text { Proportion non-White } \\
\text { patients }\end{array}$ & $7.7 \%$ & $24.3 \%$ & $3.1 \%$ & $12.0 \%$ & $23.5 \%$ & $1.3 \%$ & $3.6 \%$ \\
\hline $\begin{array}{l}\text { Proportion Hispanic } \\
\text { patients }\end{array}$ & $6.5 \%$ & $25.8 \%$ & $26.7 \%$ & $50.0 \%$ & $60.5 \%$ & $2.2 \%$ & $18.5 \%$ \\
\hline
\end{tabular}

FQHC, federally-qualified health center; RHC, rural health center.

Analysis Period: March 2016 to May 2017 (study is ongoing and will complete data collection in March 2018).

${ }^{*}$ Other Public Insurance includes publically-funded coverage sources, typically covering limited services (eg, breast and cervical cancer early detection program; title X contraceptive care) or available to specific populations (eg, Veterans Affairs and Tricare, Indian Health Service, grant programs for migrant/seasonal workers, and care for the homeless or individuals living with HIV/AIDS).

Medicaid patients, but planned to use HIT insurance support tools to assist a specific group of patients in state-sponsored coverage. A manager from health center E expressed their desire to assist these patients with insurance lapse in particular:

Because [the tools] will help us follow-up on enrollments before they are going to be due, we do not have to wait until the year's over for us to see if the patient did not renew. This is going to give us a database to build off of, to work off of, a work list if you will. Right now we do not have that. If they fall off the radar or if they did not enroll when they were supposed to, then they just fall off. There's no follow-up method. We have no easy way of doing it or tracking it. . . Because it is within [the EHR] already, it is going to make it so much easier for us because it is already there.

During the tool development period, Arm 2 clinics expressed a need for reporting tools to assist them in meeting requirements for their HRSA insurance enrollment grants, particularly regarding their efforts aiming to increase insurance support for their communities. As a requirement of HRSA O\&E grants, health centers are required to track assistance provided, yet clinics did not feel they had efficient or effective means of reporting the support that was provided. As a result of this feedback, our team enhanced the original tools by creating an additional tool function for these HRSA reporting requirements. Staff described how incorporating this new tool function was critical to streamlining the outreach and enrollment process. A supervisor at health center $\mathrm{G}$ reflected on this process:

So [HRSA reporting] used to be, manually, you go in and kind of see what you have done throughout the last months. ... I knew it was not very accurate. I felt like we were missing a lot of information.... As we're currently doing [HRSA reporting] with [the new tool], it is going to be a lot different. I should be able to pull everything from that, because it is all going to be on one thing.

\section{Quantitative Findings}

The 7 health centers (with a total of 23 study clinics) had patient populations ranging in size from 3,523 patients to 19,151 patients during the analysis period (Table 1). The rate of uninsured visits varied from $5.5 \%$ to $45.4 \%$. In all but 1 health center, more than $45 \%$ of encounters were Medicaid-insured. Race and ethnicity characteristics differed across health centers.

Clinics in 5 of 7 health centers recorded regular tool use. One health center in arm 1 (D) and 1 health center in arm 2 (E) did not record any significant tool use. Tool use was minimal in all but 1 health center (G) early on (mid-September 2016), but has increased in all but 2 health centers since then. Health center $G$ was the heaviest tool user, 
Figure 2. Tool use among arm 1 and arm 2 clinics during tool development and implementation.

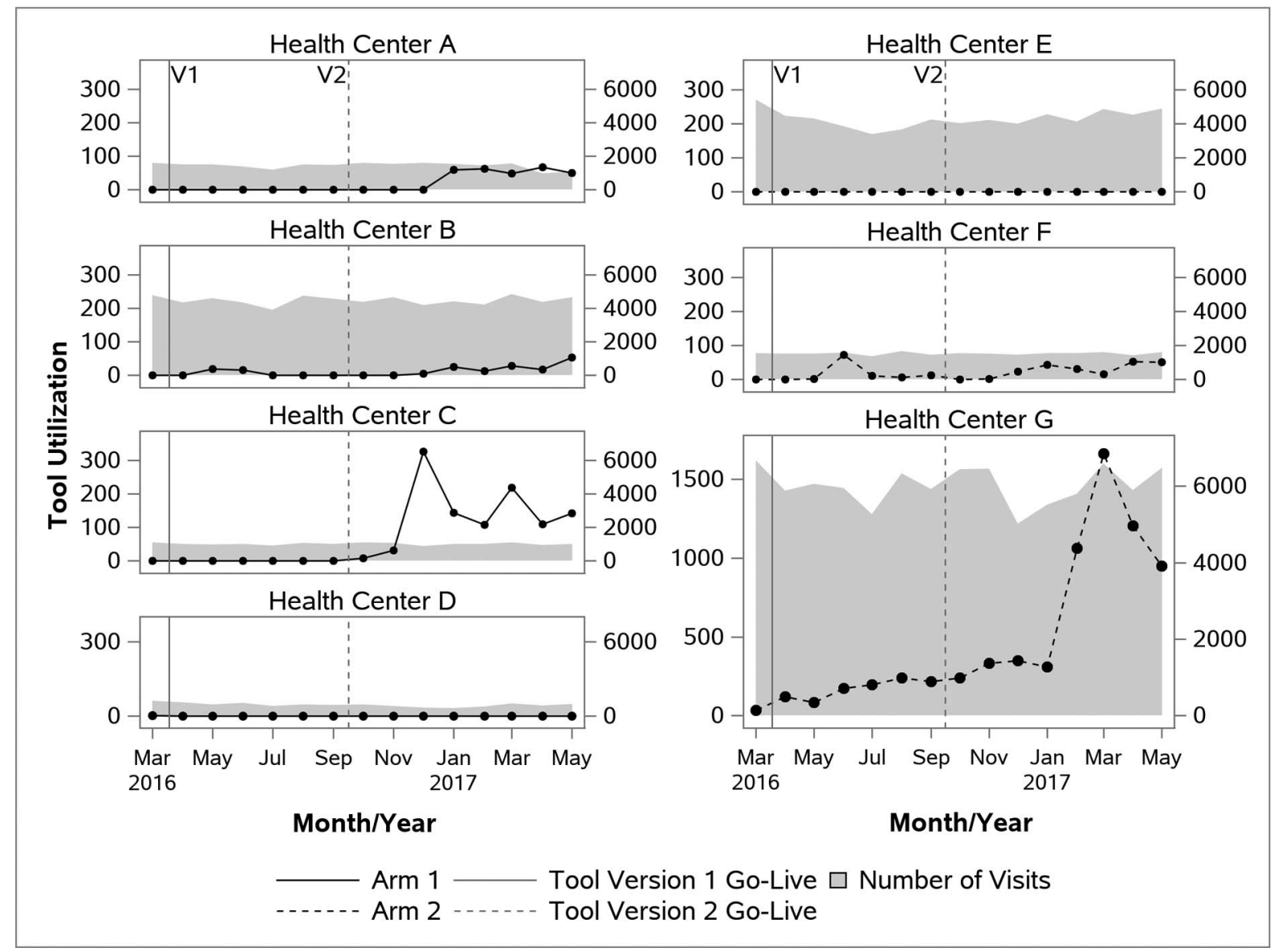

peaking at 1663 instances of tool use in March of 2017 (Figure 2), which was nearly 5-fold greater use than any other health center. In most health centers, tool use corresponded to clinic visit volume (shown in gray in Figure 2); however, health center $\mathrm{C}$ was an outlier, with a greater proportion of tool use per visit volume than other clinics. Health centers D and E, with the largest populations of Hispanic patients, demonstrated the lowest overall tool use (neither health center used the tool at all), which may suggest a link between population demographics (and perhaps insurance eligibility) and a clinic's decision to use the tools.

\section{Reflections on Supported Tool Implementation}

Arm 2 clinics experienced some shared barriers to initial tool implementation, including inadequate organizational support, staff turnover, and misunderstandings regarding tool functions. They also faced unique internal events that both hindered and supported their ability to initiate and maintain tool implementation.
At health center F, a small rural health center, $\mathrm{O} \& \mathrm{E}$ work is done by 1 eligibility specialist; shortly after tool development, this individual was reassigned. During the time in which O\&E work was being transitioned to a new person, tool use ceased until the new eligibility specialist could be trained. Preliminary qualitative analyses suggest that the lower levels of observed tool use in health center $\mathrm{F}$ are attributable to a lower clinic visit volume overall and a proportionately larger population of commercially insured patients in this clinic. In addition, staff at this clinic reported targeting tool use for patients in most need of insurance assistance rather than using the tools for all patients.

At health center $G$, a large health system, many staff members were involved in designing workflows and piloting tool use. Broad and early buy-in allowed this clinic to develop a partnership with their local Medicaid coordinated care organization in February 2017, which allowed them to start reaching out broadly to all patients whose insurance was nearing expiration, rather than just those 
patients with upcoming appointments. Having the tool available helped them strengthen this partnership, and they attributed an exponential increase in their tool use in the spring of 2017 to the partnership.

Health center E has not used the HIT tools at any time. They reflected that despite their initial desire to do so, health insurance support for their state-sponsored insurance program (for which they intended to use the tools exclusively to assist their largely immigrant population) has taken a lower priority than anticipated, and they do not expect to use the tools in the near future.

With the exception of health center E, arm 2 health centers have gradually overcome initial barriers and increased use progressively.

\section{Discussion}

This study uniquely combines a hybrid implementation-effectiveness design with principles of improvement science in a primary care setting. This design enables practice-based research teams to simultaneously assess the effectiveness of a new tool and compare levels of implementation support to optimize tool use. Here, we focused our mixedmethods analyses on early findings from tool implementation. Preliminary highlights include strong support for the tool with high levels of $\mathrm{CHC}$ enthusiasm and acceptance of the new tool, which replaces less-efficient and time-consuming processes, confirmed by early quantitative results showing that $\mathrm{CHCs}$ have been able to successfully implement the tool and are using it. Based on participant reflections and early tool use data showing greater total use among arm 2 (compared with arm 1) health centers, implementation support provided by a practice coach seems to be an effective intervention strategy to encourage adoption of novel HIT insurance assistance tools.

Early findings from this study are consistent with the large body of literature that suggests that individualized in-person support is more successful than basic educational materials for a wide variety of clinic-based interventions. ${ }^{21,22}$ Notably, the implementation support provided in our project required substantially fewer resources than successful methods of practice facilitation described in the literature ${ }^{23}$, which suggests that modest or lowintensity implementation support in the form of monthly outreach and stand-by support may be effective and important to consider for targeted primary care interventions in the future. We also found that early engagement in tool development was critical to clinic engagement, relevance of the tools, and ultimate implementation of the intervention. This finding suggests that early and frequent engagement of clinic personnel in user-centered design activities, or other similar techniques, may increase the likelihood of implementation success (as compared with rolling out a new HIT tool without engaging users in its design).

The hybrid implementation-effectiveness trial design holds promise as an effective way to assess clinic-based interventions aiming to improve clinical care quality and patient health outcomes. It shortens the time required to assess effectiveness and implementation by doing both at the same time. ${ }^{24}$ In the "real world," most interventions are implemented with little or no evaluation, which is often the case with HIT tools. This design represents an effective middle ground between quality improvement initiatives that can be done quickly and traditional research models that take a long time, but more completely document the effectiveness of an intervention before replication and dissemination. Although a hybrid implementation-effectiveness research study still takes more time and resources than a quality improvement project, it provides more depth of analysis and allows the opportunity to examine implementation and effectiveness across institutions with a high degree of scientific rigor.

\section{Limitations}

First, this study is ongoing, so the data reported here are limited to early study results. In addition, health centers are in the initial stages of spreading and standardizing workflows to use the tool consistently. Although it is too early to comprehensively assess either implementation methodology or tool effectiveness, these early findings suggest that tool use is feasible and that enhanced implementation support may be necessary to optimize use. Ongoing qualitative assessment will be critical in identifying the barriers and facilitators of increased and sustained tool use. Further, clinics self-selected for participation; thus, they are not representative of the general population of potential tool users.

In conclusion, we demonstrate promising methodologies for implementation of novel HIT tools for health insurance support at CHCs. Our results 
highlight the value of early clinic input in tool development and the potential benefit of low-intensity coaching support during HIT tool implementation. This study is one of the first to use a hybrid implementation-effectiveness design coupled with principles of improvement science in a primary care setting and demonstrates some advantages of this approach to accelerate the implementation of promising interventions in primary care.

The authors gratefully acknowledge the OCHIN practice-based research network health centers for their participation in this study.

To see this article online, please go to: http://jabfm.org/content/ 31/3/410.full.

\section{References}

1. Asplin BR, Rhodes KV, Levy H, et al. Insurance status and access to urgent ambulatory care follow-up appointments. JAMA 2005;294:1248-1254.

2. Smolderen KG, Spertus JA, Nallamothu BK, et al. Health care insurance, financial concerns in accessing care, and delays to hospital presentation in acute myocardial infarction. JAMA 2010;303:1392-1400.

3. Ayanian JZ, Weissman JS, Schneider EC, Ginsburg JA, Zaslavsky AM. Unmet health needs of uninsured adults in the United States. JAMA 2000;284:20612069.

4. DeVoe J, Fryer G, Phillips R, Green L. Receipt of preventive care among adults: insurance status and usual source of care. Am J Public Health 2003;93: 786-91.

5. Holden CD, Chen J, Dagher RK. Preventive care utilization among the uninsured by race/ethnicity and income. Am J Prev Med 2015;48:13-21.

6. Marino M, Bailey SR, Gold R, et al. Receipt of preventive services after Oregon's randomized Medicaid experiment. Am J Prev Med 2016;50:161-170.

7. Bailey SR, O'Malley JP, Gold R, Heintzman J, Marino $\mathrm{M}$, DeVoe JE. Receipt of diabetes preventive services differs by insurance status at visit. Am J Prev Med 2015;48:229-233.

8. Heintzman J, Marino M, Hoopes M, et al. Using electronic health record data to evaluate preventive service utilization among uninsured safety net patients. Prev Med. 2014;67:306-310.

9. Institute of Medicine. Care Without Coverage: Too Little, Too Late. Washington DC; 2002.

10. Wilper AP, Woolhandler S, Lasser KE, McCormick $\mathrm{D}$, Bor DH, Himmelstein DU. Health insurance and mortality in US adults. Am J Public Health 2009;99: 2289-2295.

11. Hoopes MJ, Angier H, Gold R, et al. Utilization of community health centers in Medicaid expansion and nonexpansion states, 2013-2014. J Ambul Care Manage 2016;39:290-298.

12. Angier H, Hoopes M, Gold R, et al. An early look at rates of uninsured safety net clinic visits after the Affordable Care Act. Ann Fam Med 2015;13:10-6.

13. Huguet N, Hoopes MJ, Angier H, Marino M, Holderness H, DeVoe JE. Medicaid expansion produces long-term impact on insurance coverage rates in community health centers. J Prim Care Community Health 2017;8:206-212.

14. Garfield R, Damico A, Foutz J, Kaiser Family Foundation. The Uninsured: A Primer - Key Facts about Health Insurance and the Uninsured in the Era of Health Reform. Menlo Park (CA): The Henry J Kaiser Family Foundation; 2016.

15. Garfield R., Young, K. Adults who remained uninsured at the end of 2014. Menlo Park (CA): The Henry J Kaiser Family Foundation; 2015.

16. US Department of Health and Human Services. Health centers to help uninsured Americans gain affordable health coverage. https://wayback.archiveit.org/3926/20150618191111/http://www.hhs.gov/ news/press/2013pres/07/20130710a.html. Published 2013. Accessed June 26, 2017.

17. Curran GM, Bauer M, Mittman B, Pyne JM, Stetler C. Effectiveness-implementation hybrid designs: combining elements of clinical effectiveness and implementation research to enhance public health impact. Med Care 2012;50:217-226.

18. Devoe JE, Sears A. The OCHIN community information network: bringing together community health centers, information technology, and data to support a patient-centered medical village. J Am Board Fam Med 2013;26:271-8.

19. D'Agostino RB, Jr. Propensity score methods for bias reduction in the comparison of a treatment to a non-randomized control group. Stat Med 1998;17: 2265-2281.

20. Glaser BG, Strauss A. The discovery of grounded theory: strategies for qualitative research. Chicago, IL: Aldine; 1967.

21. Geonnotti K., Taylor EF, Peikes D, et al. Engaging primary care practices in quality improvement: strategies for practice facilitators. Rockville, MD: Agency for Healthcare Research and Quality; 2015.

22. Baskerville NB, Liddy C, Hogg W. Systematic review and meta-analysis of practice facilitation within primary care settings. Ann Fam Med J 2012;10:63-74.

23. Culler SD, Parchman ML, Lozano-Romero R, et al. Cost estimates for operating a primary care practice facilitation program. Ann Fam Med 2013;11:207-11.

24. Glasgow RE, Lichtenstein E, Marcus AC. Why don't we see more translation of health promotion research to practice? Rethinking the efficacy-to-effectiveness transition. Am J Public Health 2003;93: 1261-1267. 